\title{
Estudio geológico-geotécnico para la estabilidad de taludes en el Departamento de Potosí-Bolivia
}

\author{
Geological-geotechnical study for the stability of taludes in the \\ Department of Potosí-Bolivia
}

\author{
Luis Oros Méndez \\ luis_oros@outlook.es \\ Universidad Autónoma Tomás Frías, Bolivia
}

Artículo recibido febrero 2018| Arbitrado en marzo 2018| Publicado en mayo de 2018

\section{RESUMEN}

El estudio geológico-geotécnico brinda los parámetros físico-mecánicos para el análisis de estabilidad, diseño y tratamiento de taludes. Busca prevenir y controlar los desastres causados por los deslizamientos y rotura de los taludes en una construcción civil. La determinación de análisis según los métodos propuestos SMR-RMR y FELLENIUS da una estabilización y solución teórica a los problemas conocidos ocurridos en el tramo carretero El Retiro - Puente Méndez (Progresivas 67+000-51+900), en las cuales se estudiaron las zonas críticas con problemas que dificultan el tránsito libre y seguro del movimiento automovilístico constante, como también asegurar que futuras construcciones requeridas en el tramo estudiado sean factibles y seguras. Se realizó un estudio de los factores Geológicos considerables como: la Geomorfología, la Petrografía describirá y clasificara los materiales y rocas tomando en cuenta su origen, evolución y composición, la Estratigrafía, la Geología Estructural permitió analizar el comportamiento y condiciones de la deformación existente en el área y la Geotecnia analizó las características Geomecánicas de la roca y suelo.

Palabras clave: Geológico; geotécnico; Clasificación RMR de Bieniawski; progresiva; taludes

\section{ABSTRACT}

The geological-geotechnical study provides the physical-mechanical parameters for stability analysis, design and slope treatment. It seeks to prevent and control disasters caused by landslides and breakage of the slopes in a civil construction. The determination of analysis according to the proposed methods SMR-RMR and FELLENIUS gives a stabilization and theoretical solution to the known problems that occurred in the El Retiro - Puente Méndez (Progressive $67+000$ $51+900$ ) road section, in which the Critical areas with problems that impede the free and safe traffic of the constant automobile movement, as well as ensure that future constructions required in the section studied are feasible and safe. A study of considerable Geological factors was carried out such as: Geomorphology, Petrography will describe and classify materials and rocks taking into account their origin, evolution and composition, Stratigraphy, Structural Geology allowed analyzing the behavior and conditions of the existing deformation in The area and Geotechnics analyzed the Geomechanical characteristics of rock and soil.

Key words: Geological; geotechnical; RMR Classification of Bieniawski; progressive; slopes 


\section{INTRODUCCIÓN}

La geología como ciencia, según Pedrinaci (1994), permite explicar aquellos fenómenos que se observan permanentemente en la superficie terrestre relacionados con fenómenos internos. Principalmente presenta gran interés en el conocimiento del comportamiento del planeta Tierra, sus características esenciales, los fenómenos y procesos que actúan sobre el mismo. A su vez, manifiesta Anguita (1996) que está dividida en diferentes ramas o disciplinas auxiliares que ayudan a comprender los diferentes eventos geológicos suscitados en el pasado. Actualmente, tanto Angulo (1990) como Chumacero (2004) coinciden en que ha obtenido dentro de su campo resultados satisfactorios, considerando sus métodos de estudio, que son esencialmente: la observación, la deducción, el razonamiento.

Los estudios Geológicos - Geotécnicos son indispensables para realizar un correcto cálculo, diseño, tratamiento y control relacionadas a las construcciones civiles. Estos se ejecutan con el fin de evitar problemas, deslizamientos y todo tipo de inestabilidad que afecten a los mismos. (Herrera et al. 2012; Ordaz et al. 2012).

Por otra parte, conviene tomar en cuenta lo planteado por Massad (2010) pues expresa que uno de los principales objetivos en la Geotecnia es caracterizar las propiedades de los materiales geológicos y determinar el comportamiento frente a las solicitaciones impuestas. Al momento de trabajar sobre ellas, se genera una serie de prospecciones geotécnicas en el terreno, así como una recogida selectiva de muestras para su revisión en profundidad en el laboratorio que cree un modelo geológico en función de los objetivos del estudio y de su alcance, caracterizando las propiedades de los materiales y sus discontinuidades.

Ahora bien, la Geotecnia, como una rama reciente de la geología y fundamental para ingeniería, esta surge mundialmente cuando falla la presa de St. Francis en California Meridional en el año 1928, con pérdida de vidas significativo y daños económicos. Entre los ingenieros civiles se hizo evidente la idea de que no era suficiente el diseño cuidadoso de su estructura en sí misma, para lograr una garantía de su seguridad. En este sentido, requerían de un Ingeniero Geólogo para un estudio cuidadoso de su ambiente $\mathrm{y}$, esencialmente, de los materiales sobre los cuales se asentaría la estructura. A su vez los geólogos tuvieron que familiarizarse con las exigencias ingenieriles de aquellos materiales y adquirir nociones de las funciones y comportamientos de las estructuras ingenieriles (Krynine, D. 1961). En efecto, con el trabajo realizado por Karl Terzaghi, la Mecánica de Suelos fue reconocida como una disciplina principal de la Ingeniería. Este término y el de Geología Aplicada a la Ingeniería, fueron introducidos en su libro pionero Erdbaumechanick auf Bodenphysikalischer Grundlage publicado en 1925.

La Mecánica de Rocas, comenta Villalaz (2004), fue una disciplina desarrollada por ingenieros y geólogos en la industria petrolera y minera. Esta no fue reconocida como parte de la Ingeniería Civil sino hasta 1960, en el Primer Congreso de la Sociedad Internacional de Mecánica de Rocas en Lisboa. En 1962, bajo la insistencia de la Escuela Austriaca, con Stini y Müller al frente, se desvincula de la Sociedad Internacional de Mecánica del Suelo, con la pública oposición del fundador de la ciencia geotécnica, Karl Terzaghi. Justamente en esta época, explica Sánchez Caro (2007), se producen dos hechos desafortunados: como en diciembre de 1959, falla la cimentación de la presa bóveda de Malpasset, en Francia, provocando 450 muertos. Adicionalmente, en octubre de 1963, un deslizamiento en la presa de Vajont (Italia) provoca la total destrucción, aguas abajo, de la población de Longarone, con un saldo aproximado de 2.000 muertos. Tras estudiar el desastre de Vajont, Terzaghi escribe:

(...) las curvas de frecuencia de diaclasas no pueden construirse con ningún grado de segu- 
ridad si no se realizan cientos de mediciones, si los expertos en Mecánica de Rocas no resisten la tentación de hacer pocos ensayos por razones económicas, los riesgos envueltos en las construcciones civiles en roca aumentarán fuertemente. (En: Genevois et al., 2013, y Sainz, 2013, s/n).

Entre 1973 y 1989, Bieniawski (1989) utiliza cinco parámetros básicos (resistencia a compresión simple, RQD, estado de las juntas, frecuencia de las juntas y presencia de agua) para desarrollar el RMR (Rock Mass Rating) como sistema de caracterización del terreno, presentando las primeras correlaciones entre el RMR y el módulo de deformación, permitiendo así hacer cálculos basados en teorías elásticas.

Mientras tanto, en el sur y centro de América, la Sociedad Americana de Ingenieros Civiles (ASCE) adoptó el término de Ingeniería Geotécnica en 1974. En la actualidad este último término incluye la mecánica de suelos, ingeniería de cimentaciones y mecánica de rocas, e implica una fuerte relación con la geología aplicada a la ingeniería (Celis y Colmenares, 1989; Palacio, 2013). Por esto, los países sudamericanos consideran los desastres e implementaron distintos tipos de análisis en las obras humanas como presas, embalses, túneles, carreteras, aeropuertos, minas y edificios, obteniendo resultados satisfactorios cuando previamente se determinan las condiciones geológicas-geotécnicas del terreno en el diseño y la construcción de estructuras.

Tal es el caso, como expresan Ferreras (2015) y Ochoa (2013), que para la seguridad en el diseño megaestructuras, para asegurar su estabilidad y mantenimiento, los ingenieros necesitan de la experiencia de geólogos competentes en rocas y suelos, propiedades físicas $\mathrm{y}$ químicas de las rocas, los minerales y los procesos geológicos que pueden afectarlas. Actualmente, se construyen mayores túneles, carreteras, presas, aeropuertos para grandes aviones, cortes más profun- dos para autopistas y terraplenes de mayor altura.

Concuerdan las investigaciones de Iriondo et al. (2000), Cáceres y Pinard (2004) y Mergili et al. (2015) que en Bolivia, el estudio geológico-geotécnico para realizar el diseño de una carretera es muy importante por diferentes aspectos, por ejemplo, nos indican la existencia de materiales que podemos utilizar, su distribución y accesibilidad, las propiedades de los suelos respecto del tránsito y características de los materiales en la superficie para estribos de puentes. Las características geológicas y topográficas del país muestran una diversidad de problemas activos que afectan a las construcciones civiles.

Para lo cual, vemos, a partir de lo señalado por Sanhueza y Rodríguez (2013), que la estabilidad de taludes tiene una gran importancia, ya que los deslizamientos son uno de los procesos geológicos más destructivos que afectan a los humanos causando muertes y daños en construcciones civiles. Sin embargo, es importante acotar lo expresado por Cornejo et al. (2018), pues pocos son conscientes de su importancia considerando que un porcentaje de los deslizamientos son evitables, si el problema se identifica con anterioridad y se toma medidas de prevención o control. Las zonas montañosas de altos topográficos del país son susceptibles a sufrir problemas de deslizamientos, ya que se reúnen elemen-tos importantes para su ocurrencia como son: la topografía, sismicidad, lluvias intensas, meteorización, reactivación de fallas, entre otros.

En razón, el análisis de la estabilidad de taludes es un problema clásico en la mecánica de rocas y suelos. Dichos estudios se efectúan con la finalidad de alargar su vida. Por esta razón, la estabilidad de taludes se emplea a través de un enfoque probabilístico desde inicios de los años 70. Los análisis pueden llevarse por dos métodos: el método de equilibrio límite, referido por Bojorque (2011) y el método de elementos finitos, citado por Sanz (2015). Estos métodos establecen 
propuestas de estudios novedosos que avanzarán de la mano con la tecnología disponible.

Actualmente, siguiendo de nuevo a Sanhueza y Rodríguez (2013), la mayoría de los análisis de estabilidad se basan en los estudios de equilibrio límite donde se considera la relación de esfuerzos deslizantes y resistivos a lo largo de una superficie dada. Sin embargo, en los últimos tiempos, se ha incrementado significativamente el uso de elementos finitos, considerando la división de un cuerpo en formas geométricas simples o elementos interconectados entre sí para resolver de forma aproximada el conjunto, la principal ventaja de este método es que considera las relaciones esfuerzodeformación, por lo que una rotura no se da de forma instantánea sino que es un proceso continuo. En particular el siguiente trabajo de investigación tomará en cuenta con mayor prioridad todos los estudios que engloben a la estabilidad de taludes.

Mier (2001) muestra la aplicación de métodos de ingeniería geotécnica para la estabilización afectada por fallas presentes en la zona, mejorando el libre tránsito en la carretera. Por su parte, Damaso (2009) presenta una alternativa importante y significativa con la finalidad de minimizar los accidentes de tránsito basándose en el estudio de los parámetros geológicosgeotécnicos que influyen en la estabili- zación de taludes en cortes profundos, evaluando los macizos rocosos y de suelos con la clasificación meto-dológica de Bieniawski y ejecutando paralelamente clasificaciones del macizo por el método de BARTON. Finalmente, Zambrano (2010) expone la importancia de enfocar los estudios de la geología y geotecnia aplicados a carreteras, con la finalidad de mejorar las condiciones de transitabilidad, enfocando el análisis geomecánico, basado en el comporta-miento del macizo rocoso, según los diferentes juegos de juntas o discontinuidades que muestran el grado de estabilidad.

En definitiva, considerando los constantes problemas que afectan a las carreteras y otros tipos de construcciones civiles causados por diferentes eventos naturales, geológicos, climáticos, entre otros, se pudo evidenciar la inestabilidad geológica del tramo a estudiar y con base a los problemas críticos que enfrenta el área se planteó el presente trabajo.

\section{MATERIALES Y MÉTODOS}

La zona de estudio se encuentra ubicada en el Departamento de Potosí, Provincia Cornelio Saavedra del Municipio de Betanzos, carretera principal asfaltada Potosí-Chuquisaca, con referencia menor (el Retiro-Puente Méndez). Las progresivas exactas del estudio se encuentran en la Tabla 1 realizada por Oros (2017).

Tabla 1. Progresiva

\begin{tabular}{cclccc}
\hline Punto & Progresiva & Sector & $\begin{array}{c}\text { Coordinada } \\
\text { Norte }\end{array}$ & $\begin{array}{c}\text { Coordenada } \\
\text { Este }\end{array}$ & Elevación \\
\hline $\mathbf{1}$ & $67+000$ & El Retiro-Otuyo & 7841657,413 & 267062,803 & 2564,20 \\
$\mathbf{2}$ & $51+900$ & $\begin{array}{l}\text { Millares - Abra San } \\
\text { Miguel }\end{array}$ & 7853608,553 & 269792,996 & 2455,36 \\
\hline
\end{tabular}

El tramo carretero se encuentra vinculado con las capitales de los departamentos de Potosí y Chuquisaca, a través de la carretera troncal de primer orden con una distancia de 156,4 km entre

En cuanto a la infraestructura cercana de la zona de estudio, las comunidades ambas capitales. Las progresivas de estudio se encuentran aproximadamente a 81 y $96 \mathrm{~km}$ de la capital del departamento de Potosí.

adyacentes al tramo carretero cuentan con los servicios básicos como luz y agua. Se 
observan sembradíos y construcciones de viviendas aptas para el vivir, tomando como punto central la región de Millares, la cual cuenta con un amplio comercio diario, mercado, escuela, cancha deportiva y centro de salud.

\section{a) Características Geológicas del área en estudio}

Geológicamente el área presenta una secuencia estratigráfica sobre la traza del camino de rocas ordovícicas sedimentarias de las formaciones capinota $y$ anzaldo compuestas de areniscas, limolitas, cuarcitas, pizarras y principalmente lutitas muy fracturadas con intenso diaclasa-miento poco resistentes a la erosión. Suprayasen a estas el material cuaternario que está representado por los depósitos coluvial, aluvial, terrazas aluviales, deslizamientos o de remoción en masa y depósitos de relleno artificial.

El tramo carretero en sus progresivas presenta rocas lutitas con un alto grado de fracturación y fisibilidad, presencia de vetillas de cuarzo de 0-10 $\mathrm{cm}$ de espesor. El contenido de sulfatos y carbonatos emplazadas en el material ordovícico en todo el tramo nos muestra a simple vista una coloración blanca con contenido húmedo en partes bajas muy abundante según la estación ocasional anual.

\section{b) Estratografía}

La secuencia litológica del tramo carretero comienza con el afloramiento de rocas Paleozoicas de edad Ordovícica, que comprende a las Formaciones Anzaldo y Capinota. El Punto 1 progresiva $67+000$ (sector entre el Retiro y Otuyo) con rocas ordovícicas lutitas, cuarcitas de la formación anzaldo con alto grado de diaclasamiento, estas se encuentran aflo-rando en faldas de los cerros Calla Orkho y Chaupi Orkho. El Punto progresiva 51+900 (sector millares y Abra San Miguel). En su integridad afloran rocas lutitas altamente fracturadas de la formación capinota, provenientes del flanco oeste del sinclinal que se centra en el sector de millares. En la parte superior de estas formaciones se observa material del sistema cuaternario, de serie del holoceno sedimentario principalmente aluvial y coluvial. En la Tabla 2 Oros (2017) resume litoestratigráfico, el cual indica la edad cronológica de las distintas formaciones geológicas, así como su designación litológica para los fines de investigación tomando en cuenta solo la secuencia estratigráfica de los sistemas por donde pasa el tramo de estudio.

Tabla 2. Litro-estratografía del Tramo

\begin{tabular}{ccccl}
\hline Era & Sistema & Época & $\begin{array}{c}\text { Ciclo } \\
\text { sedimentario }\end{array}$ & \multicolumn{1}{c}{ Formación } \\
\hline Cenozoico & Cuaternario & Holoceno & Andino II & Qcl, Qal, Qta, Qdm, Qar \\
Paleozoico & Ordovícico & Inf - Med & Tacsariano & $\begin{array}{l}\text { Formación Anzaldo (Oan) } \\
\text { Formación Capinota (Ocp) }\end{array}$ \\
\hline
\end{tabular}

\section{c) Geología Estructural}

Estructuralmente la zona de estudio presenta deformaciones como, plegamientos, fallas, diaclasamientos. Igualmente, la presencia de unidades mayores como anticlinales y sinclinales. Se realizó el análisis de las condiciones tectónicas locales a través de un mapeo geológico estructural por las áreas de influencia o dominios estructurales, en el cual las progresivas se ubican en material neta- mente ordovícico. El mapeo se realizó en los afloramientos rocosos de la zona que a la vez son afectados por la gravedad de manera directa. Esta se destaca como el factor primordial de activación de fallas y derrumbes, además en la diferenciación de cizallamientos, fracturaciones, entre otros. Posteriormente se realizaron las comparaciones sistemáticas para la estabilidad de los taludes. El rumbo 
predominante de los estratos varía entre las direcciones $\mathrm{N} 10^{\circ} \mathrm{W}-\mathrm{NS}-\mathrm{NE}$ con una variación de 15을 con buzamientos que fluctúan entre 40 y $70^{\circ} \mathrm{SW}$. Debido a una gran actividad tectónica en la zona de estudio se presentan dos juegos claramente visibles de diaclasas, con rumbos promedio de N $70^{\circ}$ E y Buzamiento de $80^{\circ}$ SE. El segundo juego con rumbo de $\mathrm{S} 70^{\circ} \mathrm{E}$ y buzamiento $\mathrm{NE}$.

\section{d) Geomorfología}

El área de estudio se ubica dentro de la unidad mayor de la cordillera oriental de los andes, en específico, las progresivas de estudios se encuentran delimitadas por una sucesión fluyente de ríos. La progresiva 1: entre los ríos de Tatana, Mataca y Otuyo. La progresiva 2: entre los ríos de Jatun Mayu y más lejano el rio Pilcomayo, en el cual desembocan en su mayoría todos los ríos secundarios del área, convirtiéndolo en el principal causante de los cambios relativos del paisaje y creando variaciones fluctuantes en la altura.

El paisaje y la geomorfología que presenta el área de estudio han sido afectados por diversos procesos que modelaron y efectuaron un cambio en el relieve actual, dando lugar a la formación de áreas positivas y negativas (altos y bajos topográficos). Este es el resultado de la acción de precipitaciones pluviales sumadas a los procesos de erosión, intemperismo y meteorización esencialmente física; por lo tanto, estas dieron origen a nuevas formas marcadas en el terreno, controlada por diaclasas, fallas y diaclasas locales de origen tectónico.

Las fallas regionales dieron lugar a valles jóvenes con escarpes de mediana altura. Por otra parte, la acción eólica también es muy importante en el sector, ya que dio lugar a la formación de escarpes de pendiente alta, erosionando en forma vertical y pudiendo formar valles profundos.

En el área se observaron dos tipos de redes fluviales: la red dendrítica que nos muestra una uniformidad en todas las direcciones de las rocas y la paralela nos muestra una discontinuidad que no es muy frecuente.

\section{e) Geotectónica}

La zona de estudio se encuentra dentro de la unidad geomorfológica estructural de la cordillera oriental de los Andes centro- sur, justamente entre las fallas inversas de: lineamiento Aiquile, Chinimayu, la falla Duraznillo y la falla Huayllas (más lejana), todas estas con una dirección general N-S, N-W. Encontrándose en una sucesión de anticlinales y sinclinales alrededor del área. De esta manera, dada las características observadas en la zona de estudio hay un desarrollo activo, primordialmente por las características mencionadas anteriormente.

La tectónica regional refleja un desarrollo estructural complejo atravesadas por un dominio general $\mathrm{N} 10^{\circ} \mathrm{W}$, en este sentido se desarrollan estructuras de gran envergadura de disposición regional tales como la falla inversa el Duraznillo, y los ejes de plegamientos (anticlinales y sinclinales) que dan una clara evidencia del desarrollo compresivo en dirección $\mathrm{N}$ $60^{\circ}$ E, por otra parte se observó varias fallas direccionales en sentidos opuestos producto de procesos que se desarrollaron en posteriores etapas a la fase Oclòyica en el ciclo Tacsariano del Cámbrico Ordovícico, tales como el ciclo cordillerano, ciclo sub andino hasta llegar al ciclo sedimentario Andino II.

Tectónicamente el área permitió diferenciar lo siguiente:

Primero: Ubicada al NE del área conforma un bloque paleozoico menos deformado, pero caracterizado por constantes derrumbes y deslizamientos de gravedad.

Segundo: Ubicada al NW del área presenta variación gradual de estructuras desde anticlinales hasta sinclinales, afectados por grandes fallas inversas, muy deformados por encontrarse en una zona geológica inestable.

Tercero: Ubicada al sur del área de trabajo, presencia una zona muy afectada por los deslizamientos, derrumbes, etc. 
especialmente porque en este lugar se encuentra la denominada variante el retiro que nos muestra los comportamientos de las estructuras dada la intercepción de camino carretero desde las partes más altas.

Uno de los parámetros primordiales para el problema de activación de las fallas y cualquier inestabilidad en el camino está dada por los movimientos sísmicos, es de esta manera que se puede observar que el área del proyecto se encuentra en una zona de susceptibilidad: Por lo tanto, el área de estudio se encuentra en la zona de 0.10 - 0.12 Ao/g según el Instituto San Calixto de la ciudad de la Paz, que refiere a una zona activa y de constante efecto sísmico.

La evolución tectónica del área de estudio muestra una sucesión de varios ciclos interrumpidos por efectos geodinámicos ocurridos en el margen occidental. Las mayores deformaciones y acortamientos a gran escala datan del oligoceno terminal, como resultado de la mayor actividad subductiva de la Placa de Nazca.

\section{RESULTADOS Y DISCUSIÓN}

\section{Cálculo y Clasificación RMR de Bieniawski: Mecánica de las Rocas}

Este punto se refiere a la variedad de rocas que pueden constituir un macizo rocoso. El área de estudio presenta rocas del Ordovícico como Lutitas y cuarcitas ambas muy fracturadas y diaclasadas de las Formaciones Anzaldo y Capinota. Para el muestreo de rocas y evaluación por el método de Bieniawski se utilizaron los siguientes materiales: Cateador, brújula, huincha, flexómetro, lápices de colores, reglilla de mapeo, tablero, marcadores, lupa, pintura en aerosol, escalímetro, nivel, rugómetro, guantes y equipos de protección personal.

\section{- Punto 1, Progresiva 67+000}

Estimación en el campo de la resistencia compresiva de la roca intacta, utilizando martillo de geólogo. Esta prueba se realizó en campo (in situ), realizando golpes con el cateador con una misma fuerza sobre la cara del talud sin marcas de presencia de discontinuidades, la cual según al número de golpes y características, se pudo clasificar en la tabla específica para esta estimación.

\section{Determinación de la Resistencia Compresiva mediante el Ensayo de Compresión Simple y/o Uniaxial}

Este procedimiento se realizó inicialmente con la toma de muestras del macizo de la cara del talud orientadas respectivamente. Se obtuvieron dos bloques de muestras de aproximadamente 40 kilos cada una. Luego, se realizó el respectivo corte en la máquina cortadora, con el cual se obtuvo las probetas cuyas dimensiones de ancho, largo y alto respectivamente son $7 \mathrm{~cm} \times 7 \mathrm{~cm} \times 7 \mathrm{~cm}$ (de forma cúbica). Posteriormente, estas se llevaron al laboratorio de mecánica de rocas para realizar el ensayo de compresión uniaxial. Una vez fragmentada la probeta (ver foto Nro. 8), se realizaron los cálculos y obténción de los datos respectivos.

\section{Obtención del Índice de la Calidad de la Roca - R.Q.D. \\ El RQD se determina en el campo} mediante las familias de discontinuidades o juntas, las cuales son estratos, diaclasas y toda superficie de debilidad, la distancia de trabajo de cada estación geomecánica es de acuerdo a la accesibilidad como también al tipo de trabajo, a gran detalle o un trabajo específico, la primera familia consiste en aquella que tenga más contenido de discontinuidades, la segunda familia la de un considerado contenido de discontinuidades y la tercera familia aquella que tenga menor contenido de discontinuidades. Para lo cual, tomando en cuenta a Oros (2017) se realiza el mapeo geotécnico (in situ), obteniendo los datos y ángulos en la cara del macizo de forma cubica, o sea largo (Hz), alto (Vt) y ancho (Esp). 


\section{Espaciamiento de Juntas o Disconti- nuidades}

El espaciamiento de juntas se obtiene midiendo la abertura entre estas, por lo cual se obtiene sacando la distancia promedio de los espaciamientos más representativos y comunes visados durante el mapeo, es distancia fue medida en centímetros. Para esta progresiva, las aberturas entre las discontinuidades resultan ser de 10,2 - 5,2 - 8,1 dándonos como resultado un promedio $7,8 \mathrm{~cm}$. El resultado según la tabla correspondiente se clasifica como un macizo rocoso muy fracturado.

\section{Estado de las Discontinuidades}

En este apartado se tienen en cuenta los siguientes parámetros:

- La abertura de las juntas o discontinuidades se obtiene midiendo el espacio entre las paredes de estas, por lo cual se logra sacando la distancia promedio de los espaciamientos más representativos y comunes visados durante el mapeo, esta distancia fue medida en milímetros. Para esta progresiva, las aberturas resultan ser de $8-4-6$, dándonos como resultado un promedio $6 \mathrm{~mm}$. El resultado según la tabla correspondiente se clasifica como de Grado 1 de abertura abierta.

- Esta longitud se consigue midiendo la distancia de la junta dominante, por lo cual se obtuvo sacando un promedio de las distancias más representativas visadas durante el mapeo, esta distancia fue medida en metros. Para esta progresiva, las longitudes fueron de 5,61 - 3,80 - 4,09 dándonos como resultado un promedio $4,5 \mathrm{~m}$

- La rugosidad del macizo se logró con la ayuda del rugómetro de BARTON de una longitud de 0,14 m, esta se apoyó sobre la superficie o pared del talud y se obtuvo el perfil de rugosidad, donde la profundidad más representativa llegó a ser de $6 \mathrm{~mm}$.
- Se obtiene según el tipo de material existente entre las paredes de la discontinuidad, casi siempre más blando que el macizo rocoso, estas pueden ser abiertas o cerradas y estar rellenadas o no. Se llegó a clasificar como un tipo de relleno blando < a 5 $\mathrm{mm}$, según la tabla del rango de valores para la clasificación RMR89 se valora $=2$.

- Se llegó a clasificar según un análisis (in situ), como un tipo roca muy alterada Grado IV.

- Se clasificó según un análisis (in situ), como Ligeramente Húmedo.

\section{- Punto 2, Progresiva 51+900}

Estimación en el campo de la resistencia compresiva de la roca intacta, utilizando martillo de geólogo

Esta prueba se realizó en campo (in situ), la cual simplemente realizando golpes con el cateador con una misma fuerza sobre la cara del talud sin contenido de discontinuidades, se pudo clasificar según la tabla específica para esta estimación.

\section{Determinación de la Resistencia Compresiva mediante el Ensayo de Compresión Simple y/o Uniaxial}

Este procedimiento se realizó primera-mente con la toma de muestras del macizo de la cara del talud orientadas respectivamente, se obtuvieron dos bloques de muestras de aproximadamente 40 kilos cada una. Para posteriormente realizar el respectivo corte en la máquina cortadora, con el cual se obtuvo las probetas cuyas dimensiones de ancho largo alto respectivamente son $5 \mathrm{~cm} \times 5 \mathrm{~cm}$ x $5 \mathrm{~cm}$ (de forma cúbica). Se llevó al laboratorio de mecánica de rocas para realizar el ensayo de compresión uniaxial. Una vez fragmentada la probeta se realizaron los cálculos y obtención de los datos respectivos. El resultado según la tabla correspondiente se clasifica como Roca de resistencia muy baja. 


\section{Obtención del Índice de la Calidad de la Roca - R.Q.D.}

El R.Q.D se determina en el campo mediante las familias de discontinuidades o juntas, las cuales son estratos, diaclasas y toda superficie de debilidad, la distancia de trabajo de cada estación geomecánica es de acuerdo a la accesibilidad como también al tipo de trabajo ya sea a gran detalle o un trabajo específico, la primera familia consiste en aquella que tenga más contenido de discontinuidades, la segunda familia la que tenga un considerado contenido de discontinuidades y la tercera familia aquella que tenga menor contenido de discontinuidades, para lo cual siguiendo a Oros (2017) se realizó el mapeo geotécnico (in situ), obteniendo los datos y ángulos en la cara del macizo de forma cúbica, es decir largo (Hz), alto (Vt) y ancho (Esp).

\section{Espaciamiento de Juntas o Disconti- nuidades}

El espaciamiento de juntas se obtiene midiendo la abertura entre estas, se logra sacando la distancia promedio de los espaciamientos más representativos y comunes visados durante el mapeo, esa distancia fue medida en centímetros. Para esta progresiva, las aberturas entre las discontinuidades resultan ser de 6,1 - 4 5,5 dándonos como consecuencia un promedio $5,2 \mathrm{~cm}$. El resultado según la tabla correspondiente se clasifica como un macizo rocoso muy fracturado.

\section{Estado de las Discontinuidades}

En este apartado se tienen en cuenta los siguientes parámetros:

- La abertura de las juntas o discontinuidades se obtiene midiendo el espacio entre las paredes de estas, esto se logra sacando la distancia promedio de los espaciamientos más representativos y comunes visados durante el mapeo, esta distancia fue medida en milímetros. Para esta progresiva, las aberturas resultan ser de $4-5-6$, dándonos como resultado un promedio $5 \mathrm{~mm}$. El resultado según la tabla correspondiente se clasifica como de Grado 2 de abertura moderadamente abierta.

- Esta longitud se alcanza midiendo la distancia de la junta dominante, por lo cual se obtuvo sacando un promedio de las distancias más representativas visadas durante el mapeo, esta distancia fue medida en metros. Para esta progresiva, las longitudes fueron de 2,20 - 1,55 - 3,19 dándonos como resultado un promedio $2,3 \mathrm{~m}$. El resultado según la tabla del rango de valores para la clasificación RMR89 se valora $=2$.

- La rugosidad del macizo se obtuvo con la ayuda del rugómetro de BARTON de una longitud de 0,14 m, esta se apoyó sobre la superficie o pared del talud y se alcanzó el perfil de rugosidad, donde la profundidad más representativa llegó a ser de $9 \mathrm{~mm}$, dando como resultado entre 4 - 5. Resultado para esta progresiva según la tabla correspondiente se la clasifica como: Grado de Rugosidad 4 - ondulada, lisa.

- Se obtiene según el tipo de material existente entre las paredes de la discontinuidad, casi siempre más blando que el macizo rocoso, estas pueden ser abiertas o cerradas y estar rellenadas o no. Se llegó a clasificar como un tipo de relleno blando < a 5 $\mathrm{mm}$, según la tabla del rango de valores para la clasificación RMR89 se valora $=2$.

- Se llegó a clasificar según un análisis (in situ), como un tipo roca descompuesta Grado V

- Se clasificó según un análisis (in situ), como Ligeramente Húmedo.

\section{Determinación del Factor de Segu- ridad}

Se tomó en cuenta el análisis bidimensional por la facilidad de su aplicación. Este análisis asume cero esfuerzos o cero deformaciones en las superficies laterales del modelo, por lo tanto, para que se simulen las condiciones 
de campo se requiere que existan esas condiciones o unas muy cercanas. El empleo de análisis bidimensional se puede ampliar aplicando al modelo una carga hidrostática superficial. Por otro lado, se debe tener en cuenta que los estudios para suelo reforzado requieren considerar el refuerzo del suelo mediante elementos en tensión; estos refuerzos se pueden modelar fácilmente mediante el método de equilibrio límite, pero no ocurre lo mismo mediante los modelos de esfuerzodeformación. Para el siguiente análisis del factor de seguridad se propuso utilizar el análisis según el método de Fellenius, y una comparación con los métodos de Bishop y Jambù. Utilizando el software SLIDE y GeoSlope. Para el respectivo modelamiento y obtención del Factor de seguridad.

\section{a. Cálculo del factor se seguridad para el talud Progresiva $51+900$}

El cálculo se realizó en el talud ubicado en la parte derecha de la carretera (con sentido hacia sucre), con un diseño de software de derecha-izquierda realizandolo a una escala en metros. Se utilizó el ángulo de inclinación del talud 55ํ medido en campo, las distancia inclinada y altura del talud se obtuvo por simple función trigonométrica y la diferencia de elevaciones del pie y la corona del talud, las cuales fueron $37.5 \mathrm{~m}$. - $30.7 \mathrm{~m}$. respectivamente. El tipo de suelo clasi-ficado según estudios como arena-limosa, con un peso del agua estándar de 9,81 kN/m³. La cohesión y el ángulo de fricción para el tipo de suelo obtenida por. Para el peso específico del tipo de suelo clasificado. Valores obtenidos de las tablas generales determinadas en laboratorio según los procedimientos establecidos en las normas ASTM de la American Society for Testing and Material El diseño y elaboración de la malla de análisis nos revela un factor de seguridad por debajo de 1.0: inestable (Oros, 2017)

\section{b. Cálculo del factor de seguridad para el talud Progresiva $67+000$}

El cálculo se realizó en el talud ubicado en la parte izquierda de la carretera (con sentido hacia sucre), con un diseño de software de izquierda a derecha realizándolo a una escala en metros. Se utilizó el ángulo de inclinación del talud 57ㅇ medido en campo, la distancia inclinada y altura del talud se obtuvo por simple función trigonométrica $\mathrm{y}$ la diferencia de elevaciones del pie y la corona del talud, las cuales fueron $55 \mathrm{~m}$. $46.2 \mathrm{~m}$. respectivamente. El tipo de suelo clasificado según estudios como arenalimosa, con un peso del agua estándar de $9,81 \mathrm{kN} / \mathrm{m}^{3}$. La cohesión y el ángulo de fricción para el tipo de suelo obtenida por el peso específico del tipo de suelo clasificado. Valores obtenidos de las tablas generales determinadas en laboratorio según los procedimientos establecidos en las normas ASTM de la American Society for Testing and Material (1997). El diseño y elaboración de la malla de análisis nos revela un factor de seguridad por debajo de 1.0: inestable (Oros, 2017)

\section{CONCLUSIONES}

Se realizó con éxito el levantamiento geológico - geotécnico del área y las progresivas propuestas en el estudio, permitiéndonos conocer todas las características geológicas fundamentales para contribuir al estudio realizado.

Por otra parte, en estas líneas finales es oportuno destacar que se logró realizar la clasificación RMR de Bieniawski para macizos rocosos, los cuales muestran los siguientes resultados y valores:

- Progresiva “67+000": RQD = 26 (Roca Mala), $\mathrm{RMR}=34$ Clasificación $=\mathrm{IV}$, Descripción = Roca Mala.

- Progresiva “51+900": RQD = 9 (Roca Muy Mala), RMR = 26 Clasificación = IV, Descripción = Roca Mala.

En función a la clasificación RMR, se pudo evaluar la estabilidad de los taludes por el método SMR, dando como resultado las siguientes características:

- Progresiva "67+000": SMR = 30, Clase $=\mathrm{IV},-$ Descripción = mala, Estabilidad $=$ inestable, Tratamiento $=$ Corrección . 
- Progresiva “51+900": SMR = 35, Clase $=\mathrm{IV},-$ Descripción = mala, Estabilidad = inestable, Tratamiento $=$ Corrección . Por el método de Fellenius, se determinó el factor de seguridad, dándonos los siguientes resultados:

- En las Progresivas "67+000 y "51+900": El factor de seguridad calculado en ambas fue menor a 1.00, dando a conocer un factor de seguridad bajo e inestable.

Finalmente, con todas las características y los datos obtenidos de los diferentes métodos para cada progresiva, se pudo analizar y se ofrecieron soluciones tentativas a las problemáticas existentes diseñadas y presentadas en el anexo correspondiente.

\section{REFERENCIAS}

Anguita, F. (1996). Geología y ciencias de la Tierra: etimología y un poco de historia. Enseñanza de las Ciencias de la Tierra, 4(3), 177-180

Angulo, T (1990). Geología General. Bolivia: UATF

Bieniawski, Z. T. (1989). Engineering rock mass classifications: a complete manual for engineers and geologists in mining, civil, and petroleum engineering. John Wiley and Sons

Bojorque Iñeguez, J. (2011). Métodos para el análisis de la estabilidad de pendientes

Cáceres, F., y Pirard, E. (2004). Teledetección Geológica de la Región de San Pablo de Lipez-Bolivia. En XVI Congreso Geológico Boliviano

Celis, J., y Colmenares, J. E. (2016). Hacia una formación más fundamentada y flexible en ingeniería civil. Revista Educación en Ingeniería, 11(21), 4-8

Cornejo, L., Marchán, R., y Gines, E. (2018). Riesgo por deslizamiento en el sector de "Mal Paso", Tumbes, Perú. Manglar, 15(1), 19-26

Chumacero, E. (2004). Introducción a la Geología. Bolivia: UATF

Damaso, U. (2009). Estudio de los parámetros geológicos-geotécnicos en la estabilidad de taludes en la Variante Falda de la Queñua. Bolivia: UATF
Genevois, R., Martino, S., Prestininzi, A. (2013). La Geología es noticia 50 años de la tragedia de Vajont (Italia). Enseñanza de las Ciencias de la Tierra, 21(1), 92

Ferreras Recio, A. (2015). Estudio geológico Túnel carretera Cibao-Sur

Herrera Herbert, J., y Castilla Gómez, J. (2012). Utilización de técnicas de sondeos en estudios geotécnicos: Madrid: Laboratorio de Tecnologías Mineras

Iriondo, M., Colombo, F., y Kröhling, D. (2000). El abanico aluvial del Pilcomayo, Chaco (Argentina-BoliviaParaguay): características y significado sedimentario. Geogaceta, 28(2000), 79-82

Krynine, D. P., Judd, W. R. (1961). Principios de Geología y Geotecnia para ingenieros

Massad, F. (2010). Obras de terra: curso básico de geotecnia. Oficina de textos

Mergili, M., Marchant, C., y Moreiras, S. M. (2015). Causas, características e impacto de los procesos de remoción en masa, en áreas contrastantes de la región Andina. Cuadernos de Geografía: Revista Colombiana de Geografía, 24(2), 113-131

Mier, T. (2001) Estabilización de Taludes en la carretera Cochabamba-Santa Cruz falla en el km 135 . Bolivia: Universidad Autónoma Tomas Fría

Ochoa Tapia, M. X. (2013). Estudio geológico-geotécnico del tramo de Vía Nueva Fátima-Numbiaranga-Portachuelo de los cantones Sozoranga y Macará, provincia de Loja (Bachelor's thesis).

Ordaz, A., Chuy, T. J., Hernández-Santana, J. R., y García, J. A. (2012). División geológico-geotécnica aplicada a la zonación sísmica urbana: San Cristóbal, Cuba occidental. Cuaternario y Geomorfología, 26(1-2), 89-104

Oros, L (2017). Estudio geológicogeotécnico para la estabilidad de taludes (Tramo carretero El Retiro-Puente Méndez "Progresivas 67+000+57 +900) Departamento de Potosí. Boli- 
via: Universidad Autónoma Tomas Frías

Pedrinaci, E. (1994). Historia de la Geología como herramienta didáctica, La Enseñanza de las Ciencias de la Tierra, 2(2), 332-339

Palacio, C. (2013). Tendencias y desafíos en la formación de Ingenieros Civiles. Ingeniería y Sociedad, (6), 11-19

Sainz, A. M. C. (2013). La Geología es noticia a 50 años de la catástrofe de Vajont: Riesgos de deslizamiento en el embalse de Yesa. Enseñanza de las Ciencias de la Tierra, 21(1), 101

Sánchez Caro, F. J. (2007). Seguridad de presas: aportación al análisis y control de deformaciones como elemento de prevención de patologías de origen geotécnico. En Revista de Obras Públicas
Sanhueza Plaza, C., y Rodríguez Cifuentes, L. (2013). Análisis comparativo de métodos de cálculo de estabilidad de taludes finitos aplicados a laderas naturales. Revista de la Construcción, 12(1), 17-29

Sanz, R. V., Martí, S. S., y Orrego, A. D. (2015). Estabilidad de Taludes: Conceptos Básicos, Parámetros de Diseño y Métodos de Cálculo. Civilizate, (7), 50-54

Villalaz, C. (2004). Mecánica de suelos y cimentaciones. Editorial Limusa

Zambrano, M. (2010). Estudio geológicogeotécnico del tramo chita-río mulatoBolivia: Universidad Autónoma Frías 